\title{
Loop-to-helix transition in the structure of multidrug regulator AcrR at the entrance of the drug-binding cavity
}

Babu A. Manjasetty ${ }^{\mathrm{ab}}$, Andrei S. Halavaty ${ }^{\mathrm{cd} *}$, Chi-Hao Luan ${ }^{\mathrm{de}}$, Jerzy Osipiuk ${ }^{\mathrm{dfg}}$, Rory Mulligan $^{\text {dfg }}$, Keehwan Kwon ${ }^{\text {dh }}$, Wayne F. Anderson ${ }^{\text {cd }}$ and Andrzej Joachimiak ${ }^{\mathrm{dfg}_{*}}$

${ }^{a}$ European Molecular Biology Laboratory (EMBL), Grenoble Outstation, 71 avenue des Martyrs, F-38042 Grenoble, France.

${ }^{\mathrm{b}}$ Unit of Virus-Host Cell interactions (UVHCI), University of Grenoble Alpes, F-38042 Grenoble, France.

${ }^{c}$ Biochemistry and Molecular Genetics, Feinberg School of Medicine, Northwestern University, 303 East Chicago Avenue, Chicago, IL, 60611, United States

${ }^{\mathrm{d}}$ Center for Structural Genomics of Infectious Diseases (CSGID), 303 East Chicago Avenue, Chicago, IL, 60626, United States

${ }^{\mathrm{e}}$ High Throughput Analysis Laboratory, Northwestern University, 2205 Tech Drive, Evanston, IL 60208, United States

${ }^{\mathrm{f}}$ Computational Institute, the University of Chicago, 5735 South Ellis Avenue, Chicago, IL, 60637, United States

${ }^{\mathrm{g}}$ Structural Biology Center, Argonne National Laboratory, 9700 South Cass Avenue, Argonne, IL, 60439, United States

${ }^{\mathrm{h}}$ Infectious Diseases, J. Craig Venter Institute, Rockville, MD, United States

*Correspondence email: andrzejj@anl.gov; a-halavaty@ northwestern.edu

Keywords: Transcription regulator; TetR/AcrR; Multidrug resistance; Loop-to-helix transition Abbreviations: MDR - multidrug resistance; HTH - helix-turn-helix

\begin{abstract}
Multidrug transcription regulator AcrR from Salmonella enterica subsp. enterica serovar Typhimurium str. LT2 belongs to the tetracycline repressor family, one of the largest groups of bacterial transcription factors. The crystal structure of dimeric AcrR was determined and refined to $1.56 \AA$ resolution. The tertiary and quaternary structures of AcrR are similar to those of its homologs. The multidrug binding site was identified based on structural alignment with homologous proteins and has a di(hydroxyethyl)ether molecule bound. Residues from helices $\alpha 4$ and $\alpha 7$ shape the entry into this binding site. The structure of AcrR reveals that the extended helical conformation of helix $\alpha 4$ is stabilized by the hydrogen bond between Glu67 (helix $\alpha 4$ ) and Gln130 (helix $\alpha 7$ ). Based on the structural comparison with the closest homolog structure, the Escherichia coli AcrR, we propose that this hydrogen bond is responsible for control of the
\end{abstract}


loop-to-helix transition within helix $\alpha 4$. This local conformational switch of helix $\alpha 4$ may be a key step in accessing the multidrug binding site and securing ligands at the binding site. Solution small-molecule binding studies suggest that AcrR binds ligands with their core chemical structure resembling the tetracyclic ring of cholesterol.

\section{Introduction}

Salmonella is a bacterium that causes one of the most common enteric infections and is responsible for the widely distributed foodborne disease - salmonellosis (Herikstad et al., 2002). Salmonella enterica subsp. enterica serovar Typhimurium str. LT2 is a leading cause of human gastroenteritis, and is used as a mouse model of human typhoid fever. Salmonella usually enters the host via the oral route through ingestion of contaminated food of animal origin (Miller et al., 2010; Swartz, 2002). A variety of food products have been implicated in the transmission of multidrug-resistant Salmonella isolates between animals and humans (Alban et al., 2002; Mead et al., 1999; Scallan et al., 2011; Threlfall et al., 2003). Many studies have been carried out to shed light on the spread of multidrug-resistant Salmonella isolates and to better understand multidrug resistance in salmonellosis (Ajiboye et al., 2009; Winokur et al., 2000). However, the specific mechanisms underlying drug resistance of salmonellosis is poorly understood at the molecular level. Moreover, the treatment options for the most common foodborne infections become limited as multidrug-resistant isolates, such as Salmonella, show a significant level of resistance to the most common antimicrobial drugs (European Food Safety Authority, 2015). Therefore, the development of new antibiotics against salmonellosis is of fundamental importance and a global health priority.

The tetracycline repressor (TetR) family of transcription regulators (TFRs), named after the protein that regulates genes responsible for resistance to tetracycline, generally consists of transcription repressors. Members of the family share high similarity within N-terminal helixturn-helix (HTH) DNA-binding motif and control genes that are involved in multidrug resistance, efflux pumps, virulence and pathogenicity, catabolic pathways and biosynthesis of antibiotics in bacteria (Ramos et al., 2005). These regulators bind with high affinity to their specific operator sequences, typically $\sim 10-30$-bp in length, residing within the promotor region of gene(s) that they control. TetRs repress transcription of target genes by competing with RNA polymerase for binding to the promotor. Derepression occurs when the TetRs bind their cognate ligands resulting in reducing affinity for DNA (Yu et al., 2010). 
Several microbial drug efflux pumps belonging to different transporter families have been identified and characterized experimentally ( $\mathrm{Li}$ et al., 2007). Multidrug resistance (MDR) efflux pumps exhibit multiple functions in mediating antibiotic resistance related to maintaining bacterial physiology. The major efflux system in Salmonella is AcrAB-TolC, a tripartite multidrug efflux system belonging to the resistance nodulation-division (RND) family (Martinez et al., 2009). The expression level of $a c r A B$ is correlated with the efflux function and is regulated by specific repressors and global regulatory proteins. In particular, most repressors of the efflux pumps belong to the TetR family. Although many studies have been conducted on the regulation of efflux pump expression, much less is known about these mechanisms in the important clinical isolates. It has been shown that over expression of MDR pumps, resulting in antibiotic resistance is mainly due to mutations in specific TetR regulatory genes (Grkovic et al., 1998; Hagman and Shafer, 1995). In particular, the over expression of $a c r A B$ is due to the mutations within the repressor AcrR (Olliver et al., 2004). The over expression of acrR increases organic solvent tolerance (OST) in Escherichia coli (Lee et al., 2014) and some acrR mutations result in the resistance to ceftazidime and OST (Tavio et al., 2014; Watanabe and Doukyu, 2012). In Salmonella, an increase in the gene expression of the transcription regulator ramA can be compensated by inactivating mutations (Abouzeed et al., 2008). These results indicate that regulation of MDR efflux pumps is an important component to understanding the molecular mechanisms of antibiotic resistance (Blair et al., 2015). Several crystal structures of efflux pump MDR regulators in complex with multiple drugs have been reported (Routh et al., 2009; Yamasaki et al., 2013). The crystallographic and biochemical studies revealed that members of TFRs are comprised entirely of $\alpha$-helices and form functional homodimers. However, the possibility of forming alternative dimers and even tetramers was recently discussed for the Fad35R regulator from Mycobacterium tuberculosis (Singh et al., 2015). In the TetR regulator each subunit consists of an N-terminal domain with the HTH DNA-binding motif and a Cterminal domain responsible for dimerization and ligand binding. Structure and function analyses of several TetR proteins suggested a possible regulatory mechanism in which the biological activity of TFRs is attenuated by a ligand binding within the C-terminal domain. Comparison of the crystal structures of TetR complexed with DNA (SimR, TetR, DesT, CgmR, QacR and FadR) and those with or without ligands suggests that those small molecules stabilize TetR conformations that are less capable of binding DNA (Dover et al., 2004; Frenois et al., 2004; Le et al., 2011a; Orth et al., 2000; Schumacher et al., 2002). 
Here, we report the crystallographic analysis of the transcription regulator AcrR from Salmonella enterica subsp. enterica serovar Typhimurium str. LT2 (StAcrR). StAcrR belongs to the TetR family and resembles the tertiary and quaternary structures of its homologs. A large ligand-binding cavity was identified within the C-terminal domain based on the structural comparisons with similar TetR proteins. The access to the pocket may be controlled by conformational changes within the middle portion of helix $\alpha 4$. High-throughput fluorescencebased thermal shift (FTS), fluorescence polarization (FP) binding and competition, and in silico docking data suggest that StAcrR binds ligands similar to the tetracyclic ring of cholesterol.

\section{Materials and methods}

\subsection{Cloning, expression and purification}

The acrR gene of Salmonella enterica subsp. enterica serovar Typhimurium str. LT2 encoding transcriptional repressor AcrR (the UniProt KnowledgeBase (UniProtKB) ID Q7CR15; residues 1-217) was amplified by PCR and cloned into the pMCSG7 by the ligation independent cloning technique (Schmid-Burgk et al., 2013). The resulting plasmid was transformed into BL21(DE3)/MAGIC cells that grew in the Luria-Bertani medium supplemented with ampicillin $\left(100 \mu \mathrm{g} \mathrm{ml}^{-1}\right)$ and kanamycin $\left(50 \mu \mathrm{m} \mathrm{ml}^{-1}\right)$ to $\mathrm{OD}_{600}$ of $\sim 1.0$ at $37{ }^{\circ} \mathrm{C}$ and $200 \mathrm{rev} \mathrm{min}^{-1}$ aeration. The isopropyl $\beta$-D-1-thiogalactopyranoside was added to the cells at final $1 \mathrm{mM}$ concentration for overnight induction at $18{ }^{\circ} \mathrm{C}$ and $200 \mathrm{rev} \mathrm{min}^{-1}$. Cells were harvested $\left(15,810 \mathrm{RCF} ; 4^{\circ} \mathrm{C}\right)$, resuspended in $50 \mathrm{mM}$ HEPES lysis buffer $\mathrm{pH}$ 8.0, containing $10 \mathrm{mM}$ imidazole, $500 \mathrm{mM} \mathrm{NaCl}$, $5 \%(v / v)$ glycerol, $10 \mathrm{mM} \beta$-mercaptoethanol and $1 \mathrm{mg} \mathrm{ml}^{-1}$ lysozyme and lysed by sonication. Cell debris and soluble fraction were separated by 27,485 RCF 40 -minute centrifugation at $4{ }^{\circ} \mathrm{C}$. Supernatant was loaded on a Ni-NTA column (GE Healthcare, Piscataway, NJ), which was washed with the lysis buffer to remove non-specifically bound E. coli proteins. StAcrR was eluted with the lysis buffer containing $250 \mathrm{mM}$ imidazole. StAcrR was further purified on a sizeexclusion HILoad $^{\mathrm{TM}}$ 26/60 Superdex ${ }^{\mathrm{TM}} 200$ column (GE Healthcare, Piscataway, NJ) preequilibrated with $20 \mathrm{mM}$ HEPES buffer $\mathrm{pH} 8.0,250 \mathrm{mM} \mathrm{NaCl}, 2 \mathrm{mM}$ dithiothreitol). All purification steps were performed on the ÄKTAxpress ${ }^{\mathrm{TM}}$ system (GE Healthcare, Piscataway, $\mathrm{NJ}$ ) at $4{ }^{\circ} \mathrm{C}$. After size-exclusion chromatography, StAcrR was concentrated using an Amicon Ultra-15 concentrator (Millipore, Billerica, MA, USA) with $10 \mathrm{kDa}$ molecular weight cutoff. Protein concentration was determined from the absorbance at $280 \mathrm{~nm}$ using a NanoDrop 1000 spectrophotometer (Thermo Scientific, Hanover Park, IL, USA) and the absorbance of a $0.1 \%$ (Abs $0.1 \%\left(=1 \mathrm{~g} \mathrm{l}^{-1}\right)$ ) of 0.951 for StAcrR. Purity of StAcrR was assayed by SDS-PAGE. 
StAcrR was flash cooled in liquid nitrogen and stored in $100-\mu l$ aliquots at $-80{ }^{\circ} \mathrm{C}$ till further use.

\subsection{Crystallization, data collection and structure determination}

StAcrR was crystallized by the sitting-drop vapour diffusion technique by mixing $0.4 \mu \mathrm{l}$ of 33 $\mathrm{mg} \mathrm{ml}^{-1}$ protein and $0.4 \mu \mathrm{l}$ of $200 \mathrm{mM} \mathrm{NaCl}, 100 \mathrm{mM}$ Bis-Tris buffer $\mathrm{pH} 8.5,25 \%(w / v)$ PEG 3350 at $12{ }^{\circ} \mathrm{C}$. Crystals of StAcrR were soaked in the crystallization buffer for cryoprotection and flash cooled in liquid nitrogen for data collection at $100 \mathrm{~K}$. A single-wavelength X-ray diffraction dataset was collected on the Structural Biology Center (SBC) 19-ID beamline (Rosenbaum et al., 2006) at the Advanced Photon Source (APS), Argonne National Laboratory (ANL). Diffraction images were processed with HKL-3000 (Minor et al., 2006). The StAcrR structure was determined by molecular replacement using MOLREP (Vagin and Teplyakov, 2010) integrated into HKL-3000 and the crystal structure of the transcriptional regulator AcrR from E. coli (EcAcrR; Protein Data Bank (PDB) ID: 2qop; (Li et al., 2007)) as a template. The structure was refined with REFMAC v5.5 (Murshudov et al., 1997; Murshudov et al., 2011). The Translation/Libration/Screw (TLS) refinement was applied using each protein chain as one TLS group. Data-collection and refinement statistics are given in Table 1. Diffraction images for the deposited structure are available at the Center for Structural Genomics of Infectious Diseases website (http://www.csgid.org/csgid/pages/home; target IDP02616). Figures were made using the program PyMOL v1.7.4.1 (Schrödinger, 2010). The StAcrR structure has been deposited in the PDB with accession code 31hq.

\subsection{Molecular docking}

In silico binding analysis of dequalinium, ethidium (Et), proflavine (Pf) and rhodamine 6G (R6G) at the multidrug binding pocket of StAcrR was performed with AutoDock Vina (Trott and Olson, 2010). The selected ligands were reported in the co-crystal structures of the StAcrR homologs (PDB IDs: 3vw0 (Yamasaki et al., 2013), 3vvy (Yamasaki et al., 2013), 1qvu (Schumacher et al., 2002) and 3vvz (Yamasaki et al., 2013), respectively). For docking, a ligand and StAcrR were prepared with Python Molecular Viewer (PMV) available with the MGL tools 1.5.4 (Scripps Research Institute). Firstly, water molecules were removed from the StAcrR PDB coordinates and polar hydrogen atoms were added to the protein. The protein was treated rigid including the side chains during docking. A $30 \times 30 \times 20 \AA^{3}$ docking box was prepared to cover all binding possibilities near the drug recognition residue Glu67 of StAcrR. Secondly, the ligands were prepared for the docking. The flexibility of the ligands was applied at their torsional 
angles. Each docking run included ten modes. The best mode was selected based on the binding affinity value and also by viewing the binding orientation of a ligand with respect to the bound di(hydroxyethyl)ether molecule in the structure of StAcrR. The selected best mode was used for the next docking run. For each ligand three additional runs were carried out. PyMol v1.7.4.1 (Schrödinger, 2010) was used to visualize the results.

\subsection{Fluorescence-based thermal shift assay}

Binding of dequalinium was probed with the FTS approach, while Et, Pf and R6G were studied with the FP assay (see methods description below), since they interfered with the FTS method. The FTS assay was run in 384-well PCR plates using an Echo550 acoustic transfer robot (Labcyte, Sunnyvale, CA) for dispensing a dimethyl sulfoxide stock of ligands to assay plates that contain $10 \mu \mathrm{l}$ of a mixture of StAcrR $(1 \mu \mathrm{g})$ and $2.5 \times$ SYPRO Orange fluorescence dye (Invitrogen, Carlsbad, CA) in $100 \mathrm{mM}$ HEPES buffer $\mathrm{pH} 7.5$ and $150 \mathrm{mM} \mathrm{NaCl}$. Thermal scanning (from 10 to $80{ }^{\circ} \mathrm{C}$ at $1.5{ }^{\circ} \mathrm{C} \mathrm{min}{ }^{-1}$ ramp rate) on a real-time PCR machine CFX384 (Bio-Rad Laboratories, Hercules, CA) was coupled with fluorescence detection every $10 \mathrm{~s}$.

Dequalinium bound at $40 \mu \mathrm{M}$ that prompted us to perform dose-dependent measurements with its $2.5,5,10,25,50,75$ and $100 \mu \mathrm{M}$ concentrations. Since dequalinium belongs to a 320molecule subset of the Spectrum library (Micro Source Discovery, Gaylordsville, CT, hereafter referred as SPC2-ECH008), we tested thermal stability of StAcrR against the ligands in the SPC2-ECH008 library. The best binders were selected based on difference in melting temperature $\left(\Delta T_{\mathrm{m}}\right)$, reduction of the background reading, and shape of the melting curve. The top six hits were further subjected to a dose-dependent response analysis using their $2.5,5,10,25$, 50, 75 and $100 \mu \mathrm{M}$ concentrations. Human enolase 1 (1.2 $\mu \mathrm{g}$ in $10 \mu \mathrm{l}$ assay mixture) was used as a negative control protein and tested against the top six binders and dequalinium at 10,25 and 50 $100 \mu \mathrm{M}$ concentrations. FTS data were analyzed with the in-house ExcelFTS software. The top six hits were also probed with the in silico binding method performed as described above.

\subsection{Fluorescence polarization assay}

Two-fold dilution series of StAcrR (initial $180 \mu \mathrm{M}$ ) were used and each protein concentration was tested in triplicate. Et and R6G were assayed at $1 \mu \mathrm{M}$ each. StAcrR at $40 \mu \mathrm{M}$ was completely saturated with bound Pf (tested at 250, 125, 50, or $25 \mathrm{nM}$ ). The assay buffer was 100 mM HEPES pH 7.5 and $150 \mathrm{mM} \mathrm{NaCl}$. Measurements were performed on a Tecan Infinite M1000 plate reader (Tecan Systems Inc., San Jose, CA) at ambient temperature with excitation at $470 \mathrm{~nm}$, and emission at $550 \mathrm{~nm}$ (Pf and R6G) and $600 \mathrm{~nm}(\mathrm{Et})$. Human enolase 1 and 
diflubenzuron were used as negative controls. Binding constant $\left(K_{\mathrm{d}}\right)$ for Pf, Et and R6G were determined from the equation:

$$
y=A+B /\left(1+K_{d} / x\right) \quad(\text { eq. } 1)
$$

where $y$ is FP in $\mathrm{mP}$ units, $x$ - concentration of a protein, $A-\mathrm{FP}$ of free $\mathrm{Pf}$, Et or R6G and $B-$ maximum magnitude of binding response.

The top six binders of StAcrR identified from the FTS analysis were subjected to a competition FP analysis in the presence of $100 \mathrm{nM}$ Pf using 4, 10 and $20 \mu \mathrm{M}$ StAcrR. Samples were incubated at room temperature for $30 \mathrm{~min}$ before reading. Assay was run at room temperature. This assay allowed estimation of the half maximal inhibitory concentration $\left(\mathrm{IC}_{50}\right)$, i.e. relative binding affinity, for the top six hits.

\section{Results and discussion}

\subsection{Overall structure of StAcrR}

The $P 2_{1}$ asymmetric unit of the StAcrR crystal is comprised of the chains $A$ and $B$ that form a homodimer (Fig. 1). The two chains of StAcrR are quite similar with root-mean-square (r.m.s.) deviations of $0.25 \AA$ over $209 \mathrm{C} \alpha$ atoms. The overall dimeric structure exhibits the ' $\Omega$ ' (omega)shaped form (Yu et al., 2010), which is typical to the TetR family. Each monomer has two domains: an N-terminal HTH DNA-binding domain (Met1-Lys55) and a C-terminal multidrugbinding domain (Ser56-Ala210). The interactions between the domains involve residues from helix $\alpha 1 \mathrm{~b}$ and residues from helices $\alpha 4$ and $\alpha 6$. Helix $\alpha 1$ is bent at Ala9 creating two sub-helices $\alpha 1 \mathrm{a}$ and $\alpha \mathrm{lb}$ (Fig. 1). The bending of helix $\alpha 1$ is not observed in any structures of the TetR family solved to date although significantly longer helix $\alpha 1$ was reported for the QacR (PDB ID: 1jt0; (Schumacher et al., 2002)), DesT (PDB ID: 31sj; (Miller et al., 2010)) and CgmR (PDB ID: 2yvh; (Itou et al., 2010)) structures. The length of helix $\alpha 1$ can vary from 12 to 24 residues. Non-helical N-terminal extension (28 residues) of helix $\alpha 1$ has also been reported for a TetRlike repressor SimR from Streptomyces antibioticus (SaSimR) that was shown to interact with the minor grove of bound DNA (PDB ID: 3zql; (Le et al., 2011a)). The bending of helix $\alpha 1$ in StAcrR may contribute to the stabilization of a possible StAcrR-DNA complex. In particular, residues Arg3, Lys4, Lys6 and Gln7 are near the HTH DNA-binding motif and, thus, may contribute to the protein-DNA interactions. These residues also contribute to a strong electropositive surface potential of the N-terminal region and HTH motif (Fig. 2a). Arg45 from the recognition helix $\alpha 3$ of StAcrR is conserved across close homologs in the AcrR subfamily 
and most likely is involved in interactions with the nucleotide bases. Tyr49 from helix $\alpha 3$ is also conserved, and the distance between two adjacent Tyr49 in a regulator's dimer is typically used to determine whether the DNA recognition helices are in a DNA-bound or DNA-unbound form. In StAcrR, this distance (between the $\mathrm{C} \alpha$ atoms of both Tyr49) is $39.7 \AA$, greater than that of the DNA-bound form ( $34.0 \AA$; a distance between two major grooves of DNA) yet shorter than the DNA-free form (which is either ligand-bound or apo forms). This distance slightly varies in the TetR-DNA complex structures. For example, the two Tyr42 are $34.8 \AA$ apart in the E. coli TetR complex with 15-bp DNA oligonucleotide (PDB ID: 1qpi; (Orth et al., 2000)), while two equivalent Phe41 are $36.8 \AA$ apart in the Thermotoga maritima transcriptional regulator TM1030 complexed with 24-bp DNA oligonucleotide (PDB ID: 4i6z) (Fig. 2b). Binding of tetracycline to the E. coli TetR displaces helices $\alpha 4$ and $\alpha 6$ that consequently drag its DNA-recognition helices a3 $3 \AA$ further apart and, thus, disrupting the protein-DNA interactions (Orth et al., 2000). The Tyr-to-Tyr distance varies greatly (from 37.0 to $63.4 \AA$ ) in known apo or ligand-bound TFR structures (Cuthbertson and Nodwell, 2013; Yu et al., 2010). Thus, the StAcrR structure appears to represent one of the several possible conformations between apo and ligand-bound forms of TFRs.

The C-terminal domain is comprised of helices $\alpha 4$ through $\alpha 9$ (Fig. 1). Helix $\alpha 4$ is well-ordered and relatively straight. Helices $\alpha 5, \alpha 6$ and $\alpha 7$ form a triangle like arrangement, which is found in other known TFR structures (Ramos et al., 2005). Helices $\alpha 8$ and $\alpha 9$ contribute to the dimerization interface forming four-helix bundle (Fig. 1). The StAcrR dimer buries $\sim 2,020 \AA^{2}$ surface from each monomer as estimated by the PISA server (http://www.ebi.ac.uk/pdbe/pisa/; (Krissinel and Henrick, 2007)).

\subsection{StAcrR multidrug-binding cavity}

Similar to the structurally characterized TetR family regulators, the C-terminal domain of StAcrR contains quite large ligand-binding cavity $\left(\sim 2,400 \AA^{3}\right.$; estimated with PDBsum; (de Beer et al., 2014) that is entirely contained within a single subunit. In contrast, SaSimR binds bulky simocyclinone D8 (PDB ID: 2y30; (Le et al., 2011b)), a potent DNA gyrase inhibitor, and its biosynthetic intermediate, simocyclinone C4 (PDB ID: 2y31; (Le et al., 2011b), using residues from the two subunits of the $S a S i m R$ dimer. This binding mode is possible due to the presence of a 40-residue insertion within the C-terminal domain of $\mathrm{SaSimR}$; this insertion is also involved in dimerization of SaSimR. The insertion is absent between helices $\alpha 8$ and $\alpha 9$ of StAcrR. The StAcrR multidrug-binding pocket had an extra electron density, which was interpreted as a 
di(hydroxyethyl)ether molecule (abbreviated as PEG in the StAcrR crystal structure coordinates), present in the crystallization buffer (Fig. 1). Surface representation of the StAcrR dimer shows the cavity is widely open to the solvent and is accessible to ligands through an entry located between helices $\alpha 4$ and $\alpha 7$ (Fig. 2a). In the StAcrR dimer a solvent accessible tunnel connects one ligand-binding cavity with another (not shown) similarly to one found in the crystal structure of a putative transcriptional regulator YfiR from Bacillus subtilis (PDB ID: 1rkt; (Rajan et al., 2006)). The surface of the cavity is composed of aromatic residues (Phe114 and Trp63), hydrophobic residues (Ile70, Leu93, Ile92, Ile96, Met110, Leu133, Cys134, Ser137, Ile141, Met167, Ile171 and Met175) and polar residues (Glu67, Glu74, Gln130, Glu136, Arg106, Arg140 and Arg168) (Fig. 2c). A localized negative potential patch is clearly observed at the entrance of the cavity, while the visible portion of the pocket itself has positively charged and neutral patches (Fig. 2a). This charge distribution suggests that StAcrR may bind ligands that combine neutral and charged moieties. Binding of positively charged Et, Pf and R6G by EcAcrR and EcAcrR-Glu67Ala mutant has been examined (Su et al., 2007) and it clearly showed the abolishment of the binding of Pf, Et and R6G by the Glu67Ala mutant of EcAcrR (Li et al., 2007; Su et al., 2007). The residues within the drug-binding pocket are conserved between $E c$ AcrR and StAcrR implying that these regulators may interact with structurally, yet potentially chemically diverse, ligands. In silico dockings of dequalinium, Et, Pf, R6G to StAcrR imply that the pocket is capable of accommodating these compounds (Fig. 3). The best binding mode for each ligand was identified based on its matching position with a PEG molecule bound at the multidrug-binding site of StAcrR. The AutoDock Vina-derived binding affinities were -9.3 (rhodamine 6G), -8.8 (dequalinium), -8.4 (ethidium) and -7.0 (proflavine) kcal/mol. The amino groups of the probed molecules are at an interacting distance with the carboxyl group of Glu67 (not shown) suggesting that Glu67 has a critical role in binding of a particular compound and is consistent with the ligand binding properties of the EcAcrR homolog. In addition to Glu67, the different cluster of residues that were involved in interacting with compounds indicates that multidrug binding nature of the pocket.

\subsection{Known cancer, antiviral, antimicrobial and neurological disorder drugs bind StAcrR; an} FTS study

The mean $T_{\mathrm{m}}$ of StAcrR was $57.5{ }^{\circ} \mathrm{C}$ and it increased by $\sim 1.6{ }^{\circ} \mathrm{C}$ in the presence of $40 \mu \mathrm{M}$ dequalinium. Below $10 \mu \mathrm{M}$, dequalinium did not notably change $T_{\mathrm{m}}$ of StAcrR suggesting not very specific protein-ligand interactions (Fig. 3a right panel). On the other hand, dequalinium 
has no effect on thermal stability of a negative control protein, human enolase 1 (ENO1; Fig. 3a inset), implying that dequalinium may distinctively bind StAcrR.

We identified another fifteen compounds from the SPC2-ECH008 library of 320 molecules. Those increased thermal stability of StAcrR by $\sim 1.6-6.2^{\circ} \mathrm{C}$ (see the Data in Brief Table S1 and Table S2). Six of those fifteen resemble the tetracyclic ring of cholesterol. The two TetR-type transcriptional repressors, KstR and KstR2, were shown to regulate expression of several genes involved in catabolism of cholesterol in mycobacteria (Kendall et al., 2010; Kendall et al., 2007) and related actinomycetes (Van der Geize et al., 2007). The co-crystal structure of KstR2 and 3a $\alpha-H-4 \alpha(3$ '-propanoate)-7a $\beta$-methylhexahydro-1,5-indanedione (HIP)-coenzyme A (HIP-CoA) (PDB ID: 4w97; (Crowe et al., 2015)) revealed that the HIP moiety of cholesterol binds at the multidrug-binding site of every KstR2 subunit, while the CoA portion binds at the dimerization interface. The HIP-(CoA)-like structures are also present among the StAcrR hits (the Data in Brief Table S1).

The top six StAcrR binders showed no effect on thermal stability of human ENO1 (see the Data in Brief Fig. S1). Apparent increase in the StAcrR thermal stability at very low concentrations of fulvestrant, ritonavir and estramustine suggests that these compounds are distinct binders (Fig. 4 and Fig. 5). In silico calculations may support this notion with the estimated binding affinities of -9.6 (ritonavir), -8.9 (estramustine) and -8.7 (fulvestrant) $\mathrm{kcal} / \mathrm{mol}$. In contrast, prasterone (-8.5 $\mathrm{kcal} / \mathrm{mol})$, triclabendazole $(-7.2 \mathrm{kcal} / \mathrm{mol})$ and idebenone $(-6.4 \mathrm{kcal} / \mathrm{mol})$ required higher concentrations to achieved similar effects with StAcrR. However, in silico-derived affinities should be considered with caution as they only provide a guidance of how strong or weak a ligand can bind in a given calculation approach. Although binding modes for the top ligands cannot be determined or distinguished from the FTS analysis, the in silico docking data may yet provide some insights into their coordination in the multidrug-binding pocket (Fig. 4 and Fig. 5). All top compounds are positioned within the center section of the binding cavity, while parts of bulky ritonavir and estramustine occupy sites at the entry into a solvent accessible tunnel that connects the individual multidrug-binding cavities at the dimerization interface of StAcrR (Fig. 5). Based on the FTS data and the fact that StAcrR does not have any further comparable smallmolecule binding pocket(s) of $\sim 2,400 \AA^{3}$, we hypothesized that the top six and perhaps the remaining hits bind in the protein's multidrug-binding site. None of the tested by FTS ligands increased thermal stability of human ENO1 further supporting the hypothesis.

\subsection{Size and chemical properties of a ligand affect its affinity to StAcrR: an FP analysis}


Pf had over 10-fold higher affinity to StAcrR than Et and R6G (Table 2 and Fig. 6) implying that its more compact 3D structure and chemical properties favour the interactions. No FP increase in the presence of Pf was observed for human ENO1 (up to $100 \mu \mathrm{M}$ ) supporting the specific nature of the StAcrR-Pf interactions. Diflubenzuron did not compete with Pf for binding (Table 2).

Relative binding affinities $\left(\mathrm{IC}_{50}\right)$ for the top six FTS ligands were determined from the competition FP assay (Table 2). Perhaps not surprisingly, comparably small-size idebenone ( $\mathrm{IC}_{50}$ of $\sim 3 \mu \mathrm{M}$; FTS rank 2) and prasterone ( $\mathrm{IC}_{50}$ of $\sim 5 \mu \mathrm{M}$; FTS rank 6 ) are the top two inhibitors of Pf. Although ritonavir ( $\left(\mathrm{IC}_{50}\right.$ of $\sim 12 \mu \mathrm{M}$; FTS rank 4$)$ is bulky, presumably threetime surplus of its polar surface area over that of Pf has greater inhibitory effect than smaller and more hydrophobic triclabendazole ( $\mathrm{IC}_{50}$ of $\sim 26 \mu \mathrm{M}$; FTS rank 3), estarmustine $\left(\mathrm{IC}_{50}\right.$ of $\sim 18 \mu \mathrm{M}$; FTS rank 5) and dequalinium chloride ( $\mathrm{IC}_{50}$ of $\sim 23 \mu \mathrm{M}$; FTS rank 14) (Table 2). Chlorines within those compounds may contribute to higher $\mathrm{IC}_{50}$ values, while competing for positively charged patches within the multidrug-binding pocket. Fulvestrant, the number 1 FTS hit, had the highest $\mathrm{IC}_{50}$ of $\sim 40 \mu \mathrm{M}$ that may be accounted for its complex chemical composition.

The rear and top sections of the multidrug-binding pocket of StAcrR are fairly hydrophobic and partially masked by charged surface-exposed residues of helices $\alpha 4$ and $\alpha 7$; the bottom section is charged (Fig. 2c). Thus, accommodation of aliphatic moieties of some tested ligands would presumably require local structural rearrangements (see below) to access hydrophobic patches. Thus, high affinity of Pf and its strongest inhibitors may be explained by their easy access to and few-to-none structural changes around/within the pocket upon binding. Although we could not assess the FTS rank of Pf due to experimental limitations of FTS, comparing its binding affinity to that of idebenone (FTS rank 2) and prasterone (FTS rank 9) one proposes, that although Pf is a strong binder, it may not have a drastic effect on the thermal stability of StAcrR. Obviously, charged and hydrophobic moieties of a ligand play a key role in affecting the protein's thermal stability, i.e. the tertiary structure within the multidrug-binding domain and likely DNA-binding domain of StAcrR.

\subsection{Ligand binding necessitates loop-to-helix transition in StAcrR}

The search for structural homologs using the Vector Alignment Search Tool (VAST) (Gibrat et al., 1996) revealed 138 neighbours in the PDB. The closest structural homologs are EcAcrR (sequence identity 88.2\%; PDB ID: 3bcg; (Gu et al., 2008); r.m.s. deviations $1.2 \AA$ over $203 \mathrm{C} \alpha$ atoms) and transcription regulator TtgR from Pseudomonas putida (PpTtgR; sequence identity 37.4\%; PDB ID: 2uxo; (Alguel et al., 2007); r.m.s. deviations $1.9 \AA$ over $198 \mathrm{C} \alpha$ atoms). The 
overall fold is highly conserved, however, the significant structural differences are observed in the conformation of helix $\alpha 4$. In the StAcrR structure, helix $\alpha 4$ is straight and extended (Fig. 7), whereas in both crystal forms of apo $E c$ AcrR (EcAcrR-P3 ${ }_{1}$; PDB ID: $3 b c g$ (Gu et al., 2008); and EcAcrR-P222 1 ; PDB ID: 2qop; (Li et al., 2007)), helix $\alpha 4$ unwinds locally into a loop region (residues Leu65-Ile70 in EcAcrR-P222 ${ }_{1}$ and Leu65-Leu73 in EcAcrR-P3 1 that are equivalent to residues Leu65-Leu73 in StAcrR) splitting helix $\alpha 4$ into two sub-helices, $\alpha 4 a$ and $\alpha 4 b$ (Fig. 7). Similar, local unwinding of helix $\alpha 4$ along with its bending is observed in both chains of the binary tetracycline- $P p \operatorname{TtgR}$ complex structure (not shown), yet the antibiotic was modelled at the ligand-binding site of one chain only. Thus, comparison of the aforementioned structures of three homologous regulators suggests that conformation of helix $\alpha 4$ would depend on whether a regulator is in a ligand-free or ligand-bound form and the size of a ligand may determine the extent of local alterations in the structure. Additionally, in the structures of MDR regulator RamR from S. typhimurium (PDB ID: 3vvy; (Yamasaki et al., 2013)) and transcriptional repressor LfrR from M. smegmatis (PDB ID: 2v57; (Bellinzoni et al., 2009)) helix $\alpha 7$ undergoes conformational change in order to accommodate a drug, ethidium and proflavine, respectively. Thus, flexibility of helices $\alpha 4$ and $\alpha 7$ may be an important property that may be common in the MDR transcription regulators allowing them to bind ligands of different chemistry and size.

In StAcrR, the ligand recognition residue Glu67 from helix $\alpha 4$ interacts with Gln130 from helix $\alpha 7$ and, thus, stabilizes the local tertiary structure. Structural comparison of StAcrR with the two crystal forms of EcAcrR reveals that Glu67 and Gln130 adopt different side chain conformations (Fig. 7). In the EcAcrR-P222 ${ }_{1}$ crystal form, Glu67 forms the hydrogen bond with Arg106 (helix a6) whereas Glu67 in the EcAcrR-P3 $3_{1}$ lost that interaction and is solvent exposed. In the StAcrR structure, the interaction between Glu67 and Arg106 is not observed. Thus, Glu67 can participate in alternative interactions. Further, the hydrogen-bonding network of Arg 105 with the N-terminal residues Gln14 and Asp18 (helix $\alpha 1 b$ ) is completely retained in the StAcrR and EcAcrR-P222 ${ }_{1}$ structures (Fig. 7). This hydrogen bond network seems to be disrupted in the $E c$ AcrR-P $3_{1}$ though the side chain conformations of these residues have poor fit to the electron density (not shown). Taken together, we hypothesize that helices $\alpha 4$ and $\alpha 7$ of the C-terminal domain function as gatekeepers of the StAcrR multidrug-binding cavity allowing or preventing the entry of particular drugs. Apparently, the helices exhibit some level of conformational diversity, as seen in homologous repressors too, that is may be crucial for precise recognition and accommodation of specific drugs, e.g. those identified by the FTS analysis, within the multidrug-binding cavity of StAcrR. 


\section{Acknowledgements}

The CSGID project has been funded in whole or in part with Federal funds from the National Institute of Allergy and Infectious Diseases, National Institutes of Health, Department of Health and Human Services, under Contracts No. HHSN272200700058C and HHSN272201200026C. We thank Sankar Krishnna for the human ENO1 sample. The authors wish to thank members of the Structural Biology Center (SBC) at Argonne National Laboratory for their help with X-ray diffraction data collection. The operation of SBC beamlines is supported by the U.S. Department of Energy, Office of Biological and Environmental Research under contract DE-AC0206CH11357.

\section{References}

Abouzeed, Y.M., Baucheron, S., Cloeckaert, A., 2008. ramR mutations involved in effluxmediated multidrug resistance in Salmonella enterica serovar Typhimurium. Antimicrob. Agents Chemother. 52, 2428-2434.

Ajiboye, R.M., Solberg, O.D., Lee, B.M., Raphael, E., Debroy, C., Riley, L.W., 2009. Global spread of mobile antimicrobial drug resistance determinants in human and animal Escherichia coli and Salmonella strains causing community-acquired infections. Clin. Infect. Dis. 49, 365-371.

Alban, L., Olsen, A.M., Nielsen, B., Sorensen, R., Jessen, B., 2002. Qualitative and quantitative risk assessment for human salmonellosis due to multi-resistant Salmonella typhimurium DT104 from consumption of Danish dry-cured pork sausages. Prev. Vet. Med. 52, 251265.

Alguel, Y., Meng, C., Teran, W., Krell, T., Ramos, J.L., Gallegos, M.T., Zhang, X., 2007. Crystal structures of multidrug binding protein TtgR in complex with antibiotics and plant antimicrobials. J. Mol. Biol. 369, 829-840.

Bellinzoni, M., Buroni, S., Schaeffer, F., Riccardi, G., De Rossi, E., Alzari, P.M., 2009. Structural plasticity and distinct drug-binding modes of LfrR, a mycobacterial efflux pump regulator. J. Bacteriol. 191, 7531-7537.

Blair, J.M., Webber, M.A., Baylay, A.J., Ogbolu, D.O., Piddock, L.J., 2015. Molecular mechanisms of antibiotic resistance. Nat. Rev. Microbiol. 13, 42-51.

Chen, V.B., Arendall, W.B., 3rd, Headd, J.J., Keedy, D.A., Immormino, R.M., Kapral, G.J., Murray, L.W., Richardson, J.S., Richardson, D.C., 2010. MolProbity: all-atom structure validation for macromolecular crystallography. Acta Crystallogr. D Biol. Crystallogr. 66, $12-21$.

Crowe, A.M., Stogios, P.J., Casabon, I., Evdokimova, E., Savchenko, A., Eltis, L.D., 2015. Structural and functional characterization of a ketosteroid transcriptional regulator of Mycobacterium tuberculosis. J. Biol. Chem. 290, 872-882.

Cuthbertson, L., Nodwell, J.R., 2013. The TetR family of regulators. Microbiol. Mol. Biol. Rev. 77, 440-475.

de Beer, T.A., Berka, K., Thornton, J.M., Laskowski, R.A., 2014. PDBsum additions. Nucleic Acids Res. 42, D292-296.

Dover, L.G., Corsino, P.E., Daniels, I.R., Cocklin, S.L., Tatituri, V., Besra, G.S., Futterer, K., 2004. Crystal structure of the TetR/CamR family repressor Mycobacterium tuberculosis EthR implicated in ethionamide resistance. J. Mol. Biol. 340, 1095-1105. 
European Food Safety Authority, 2015. Salmonella and Campylobacter show significant levels of resistance to common antimicrobials in humans and animals.

Frenois, F., Engohang-Ndong, J., Locht, C., Baulard, A.R., Villeret, V., 2004. Structure of EthR in a ligand bound conformation reveals therapeutic perspectives against tuberculosis. Mol. Cell 16, 301-307.

Gibrat, J.F., Madej, T., Bryant, S.H., 1996. Surprising similarities in structure comparison. Curr. Opin. Struct. Biol. 6, 377-385.

Gouet, P., Courcelle, E., Stuart, D.I., Metoz, F., 1999. ESPript: analysis of multiple sequence alignments in PostScript. Bioinformatics 15, 305-308.

Grkovic, S., Brown, M.H., Roberts, M.J., Paulsen, I.T., Skurray, R.A., 1998. QacR is a repressor protein that regulates expression of the Staphylococcus aureus multidrug efflux pump QacA. J. Biol. Chem. 273, 18665-18673.

Gu, R., Li, M., Su, C.C., Long, F., Routh, M.D., Yang, F., McDermott, G., Yu, E.W., 2008. Conformational change of the AcrR regulator reveals a possible mechanism of induction. Acta Crystallogr. Sect. F: Struct. Biol. Cryst. Commun. 64, 584-588.

Hagman, K.E., Shafer, W.M., 1995. Transcriptional control of the mtr efflux system of Neisseria gonorrhoeae. J. Bacteriol. 177, 4162-4165.

Herikstad, H., Motarjemi, Y., Tauxe, R.V., 2002. Salmonella surveillance: a global survey of public health serotyping. Epidemiol. Infect. 129, 1-8.

Itou, H., Watanabe, N., Yao, M., Shirakihara, Y., Tanaka, I., 2010. Crystal structures of the multidrug binding repressor Corynebacteriumglutamicum $\mathrm{CgmR}$ in complex with inducers and with an operator. J. Mol. Biol. 403, 174-184.

Kendall, S.L., Burgess, P., Balhana, R., Withers, M., ten Bokum, A., Lott, J.S., Gao, C., UhiaCastro, I., Stoker, N.G., 2010. Cholesterol utilization in mycobacteria is controlled by two TetR-type transcriptional regulators: $k s t R$ and $k s t R 2$. Microbiology 156, 1362-1371.

Kendall, S.L., Withers, M., Soffair, C.N., Moreland, N.J., Gurcha, S., Sidders, B., Frita, R., Ten Bokum, A., Besra, G.S., Lott, J.S., Stoker, N.G., 2007. A highly conserved transcriptional repressor controls a large regulon involved in lipid degradation in Mycobacterium smegmatis and Mycobacterium tuberculosis. Mol. Microbiol. 65, 684699.

Kisker, C., Hinrichs, W., Tovar, K., Hillen, W., Saenger, W., 1995. The complex formed between Tet repressor and tetracycline-Mg2+ reveals mechanism of antibiotic-resistance. J. Mol. Biol. 247, 260-280.

Krissinel, E., Henrick, K., 2007. Inference of macromolecular assemblies from crystalline state. J. Mol. Biol. 372, 774-797.

Le, T.B., Schumacher, M.A., Lawson, D.M., Brennan, R.G., Buttner, M.J., 2011a. The crystal structure of the TetR family transcriptional repressor SimR bound to DNA and the role of a flexible N-terminal extension in minor groove binding. Nucleic Acids Res. 39, 94339447.

Le, T.B., Stevenson, C.E., Fiedler, H.P., Maxwell, A., Lawson, D.M., Buttner, M.J., 2011b. Structures of the TetR-like simocyclinone efflux pump repressor, SimR, and the mechanism of ligand-mediated derepression. J. Mol. Biol. 408, 40-56.

Lee, J.O., Cho, K.S., Kim, O.B., 2014. Overproduction of AcrR increases organic solvent tolerance mediated by modulation of SoxS regulon in Escherichia coli. Appl. Microbiol. Biotechnol. 98, 8763-8773.

Li, M., Gu, R., Su, C.C., Routh, M.D., Harris, K.C., Jewell, E.S., McDermott, G., Yu, E.W., 2007. Crystal structure of the transcriptional regulator AcrR from Escherichia coli. J. Mol. Biol. 374, 591-603. 
Martinez, J.L., Sanchez, M.B., Martinez-Solano, L., Hernandez, A., Garmendia, L., Fajardo, A., Alvarez-Ortega, C., 2009. Functional role of bacterial multidrug efflux pumps in microbial natural ecosystems. FEMS Microbiol. Rev. 33, 430-449.

Mead, P.S., Slutsker, L., Dietz, V., McCaig, L.F., Bresee, J.S., Shapiro, C., Griffin, P.M., Tauxe, R.V., 1999. Food-related illness and death in the United States. Emerg. Infect. Dis. 5, 607-625.

Miller, D.J., Zhang, Y.M., Subramanian, C., Rock, C.O., White, S.W., 2010. Structural basis for the transcriptional regulation of membrane lipid homeostasis. Nat. Struct. Mol. Biol. 17, 971-975.

Minor, W., Cymborowski, M., Otwinowski, Z., Chruszcz, M., 2006. HKL-3000: the integration of data reduction and structure solution - from diffraction images to an initial model in minutes. Acta Crystallogr. D Biol. Crystallogr. 62, 859-866.

Murshudov, G.N., Vagin, A.A., Dodson, E.J., 1997. Refinement of macromolecular structures by the maximum-likelihood method. Acta Crystallogr. D Biol. Crystallogr. 53, 240-255.

Murshudov, G.N., Skubak, P., Lebedev, A.A., Pannu, N.S., Steiner, R.A., Nicholls, R.A., Winn, M.D., Long, F., Vagin, A.A., 2011. REFMAC5 for the refinement of macromolecular crystal structures. Acta Crystallogr. D Biol. Crystallogr. 67, 355-367.

Olliver, A., Valle, M., Chaslus-Dancla, E., Cloeckaert, A., 2004. Role of an acrR mutation in multidrug resistance of in vitro-selected fluoroquinolone-resistant mutants of Salmonella enterica serovar Typhimurium. FEMS Microbiol. Lett. 238, 267-272.

Orth, P., Schnappinger, D., Hillen, W., Saenger, W., Hinrichs, W., 2000. Structural basis of gene regulation by the tetracycline inducible Tet repressor-operator system. Nat. Struct. Biol. 7, 215-219.

Rajan, S.S., Yang, X., Shuvalova, L., Collart, F., Anderson, W.F., 2006. Crystal structure of YfiR, an unusual TetR/CamR-type putative transcriptional regulator from Bacillus subtilis. Proteins 65, 255-257.

Ramos, J.L., Martínez-Bueno, M., Molina-Henares, A.J., Terán, W., Watanabe, K., Zhang, X., Gallegos, M.T., Brennan, R., Tobes, R., 2005. The TetR family of transcriptional repressors. Microbiol. Mol. Biol. Rev. 69, 326-356.

Rosenbaum, G., Alkire, R.W., Evans, G., Rotella, F.J., Lazarski, K., Zhang, R.G., Ginell, S.L., Duke, N., Naday, I., Lazarz, J., Molitsky, M.J., Keefe, L., Gonczy, J., Rock, L., Sanishvili, R., Walsh, M.A., Westbrook, E., Joachimiak, A., 2006. The Structural Biology Center 19ID undulator beamline: facility specifications and protein crystallographic results. J. Synchrotron Radiat. 13, 30-45.

Routh, M.D., Su, C.C., Zhang, Q.J., Yu, E.W., 2009. Structures of AcrR and CmeR: Insight into the mechanisms of transcriptional repression and multi-drug recognition in the TetR family of regulators. Biochim. Biophys. Acta 1794, 844-851.

Scallan, E., Hoekstra, R.M., Angulo, F.J., Tauxe, R.V., Widdowson, M.A., Roy, S.L., Jones, J.L., Griffin, P.M., 2011. Foodborne illness acquired in the United States-major pathogens. Emerg. Infect. Dis. 17, 7-15.

Schmid-Burgk, J.L., Schmidt, T., Kaiser, V., Honing, K., Hornung, V., 2013. A ligationindependent cloning technique for high-throughput assembly of transcription activatorlike effector genes. Nat. Biotechnol. 31, 76-81.

Schrödinger, L., 2010. PyMOL.

Schumacher, M.A., Miller, M.C., Grkovic, S., Brown, M.H., Skurray, R.A., Brennan, R.G., 2002. Structural basis for cooperative DNA binding by two dimers of the multidrugbinding protein QacR. EMBO J. 21, 1210-1218.

Singh, A.K., Manjasetty, B., Balasubramani, G.L., Koul, S., Kaushik, A., Ekka, M.K., Singh, V., Kumaran, S., 2015. Crystal structure of Fad35R from Mycobacterium tuberculosis H37Rv in the apo-state. PLoS One 10, e0124333. 
Su, C.C., Rutherford, D.J., Yu, E.W., 2007. Characterization of the multidrug efflux regulator AcrR from Escherichia coli. Biochem. Biophys. Res. Commun. 361, 85-90.

Swartz, M.N., 2002. Human diseases caused by foodborne pathogens of animal origin. Clin. Infect. Dis. 34 Suppl. 3, S111-S122.

Tavio, M.M., Aquili, V.D., Vila, J., Poveda, J.B., 2014. Resistance to ceftazidime in Escherichia coli associated with AcrR, MarR and PBP3 mutations and overexpression of sdiA. J. Med. Microbiol. 63, 56-65.

Threlfall, E.J., Fisher, I.S., Berghold, C., Gerner-Smidt, P., Tschape, H., Cormican, M., Luzzi, I., Schnieder, F., Wannet, W., Machado, J., Edwards, G., 2003. Antimicrobial drug resistance in isolates of Salmonella enterica from cases of salmonellosis in humans in Europe in 2000: results of international multi-centre surveillance. Euro Surveill. 8, 4145.

Trott, O., Olson, A.J., 2010. AutoDock Vina: improving the speed and accuracy of docking with a new scoring function, efficient optimization, and multithreading. J. Comput. Chem. 31, 455-461.

Vagin, A., Teplyakov, A., 2010. Molecular replacement with MOLREP. Acta Crystallogr. D Biol. Crystallogr. 66, 22-25.

Van der Geize, R., Yam, K., Heuser, T., Wilbrink, M.H., Hara, H., Anderton, M.C., Sim, E., Dijkhuizen, L., Davies, J.E., Mohn, W.W., Eltis, L.D., 2007. A gene cluster encoding cholesterol catabolism in a soil actinomycete provides insight into Mycobacterium tuberculosis survival in macrophages. Proc. Natl. Acad. Sci. U S A 104, 1947-1952.

Watanabe, R., Doukyu, N., 2012. Contributions of mutations in acrR and marR genes to organic solvent tolerance in Escherichia coli. AMB Express 2, 58.

Winokur, P.L., Brueggemann, A., DeSalvo, D.L., Hoffmann, L., Apley, M.D., Uhlenhopp, E.K., Pfaller, M.A., Doern, G.V., 2000. Animal and human multidrug-resistant, cephalosporinresistant salmonella isolates expressing a plasmid-mediated CMY-2 AmpC betalactamase. Antimicrob. Agents Chemother. 44, 2777-2783.

Yamasaki, S., Nikaido, E., Nakashima, R., Sakurai, K., Fujiwara, D., Fujii, I., Nishino, K., 2013. The crystal structure of multidrug-resistance regulator RamR with multiple drugs. Nat. Commun. 4, 2078.

Yu, Z., Reichheld, S.E., Savchenko, A., Parkinson, J., Davidson, A.R., 2010. A comprehensive analysis of structural and sequence conservation in the TetR family transcriptional regulators. J. Mol. Biol. 400, 847-864.

Fig. 1. Ribbon diagram of the StAcrR homodimeric structure. The left monomer is shown in the two different colors to represent the N-terminal HTH DNA-binding domain (blue) and a Cterminal small-molecule binding domain (green). The right monomer is shown in orange. The di(hydroxyethyl)ether (PEG) (depicted as spheres and colored according to atoms: carbon (grey) and oxygen (red)) bound to the C-terminal domain's multidrug-binding site of both monomers is shown. Arg45, important for DNA binding, and Glu67, important for drug binding are shown in stick representation. Gln130 that interacts with Glu67 is shown. Distance between the two Tyr49 
is used to measure the distance between the DNA recognition helices to represent DNA-bound, apo and ligand-bound forms of a transcriptional regulator.

Fig. 2. The HTH DNA-binding grove and multidrug-binding pocket. (a) Side view of the electrostatic surface potential of StAcrR with the di(hydroxyethyl)ether molecule (PEG; carbon atoms (green); oxygen atoms (red)) bound at the ligand-binding pocket. (b) Left panel, side view of the superimposed structures of StAcrR (orange; PEG is in stick model (orange)), the Thermotoga maritima transcriptional regulator TM1030 (blue) with 24-bp DNA (yellow) oligonucleotide and the TM1030 protein (green; PDB ID: 2iek) with hexaethylene glycol (P6G; green sticks) bound. Right panel, side view of the superimposed structures of StAcrR (as in the left panel), the E. coli TetR (green; PDB ID: 1qpi) with 15-bp DNA (yellow) oligonucleotide and the E. coli TetR (grey; PDB ID: 2tct; (Kisker et al., 1995)) with 7-cholortetracycline (grey sticks) bound. Helices of StAcrR are labelled and corresponding Tyr (Phe41 in the 1qpi structure) residues that are used to measure the Tyr-Tyr distance in a TetR dimer are shown. Single protomer of a TetR dimer is shown only. Bottom panel, multiple sequence alignment (area of the N-terminal HTH DNA-binding domain) of the StAcrR (UniProtKB ID: Q7CR15), TM1030 (UniProtKB ID: Q9X0C0) and E. coli TetR (UniProtKB ID: P0ACT4) proteins. The C-terminal portion of the alignment has fewer conserved residues. Figure was generated with ESPript v2.2 (Gouet et al., 1999) using SimilarityGlobalScore of 0.7, SimilarityDiffScore of 0.5 and SimilarityType Risler. (c) Key residues (stick representation) that comprise the multidrug ligand-binding pocket. The $3 \sigma$ OMIT map (magenta mesh) of PEG.

Fig. 3. AutoDock Vina docking results for dequalinium and the FTS-derived dose-dependent denaturation profile of StAcrR (a). Inset shows dose-dependent FTS data of human enolase 1 (ENO1), a negative control protein, with dequalinium. Docking results for ethidium (b), proflavine (c) and rhodamine 6G (d). Small molecules are shown in sticks representation and colored according to atoms: carbon (light grey); oxygen (red); and nitrogen (blue).

Fig. 4. Dose-dependent FTS melting curves (left) and AutoDock Vina docking results (right) for fulvestrant, idebenone and triclabendazole.

Fig. 5. Dose-dependent FTS melting curves (left) and AutoDock Vina docking results (right) for ritonavir, estramustine and prasterone.

Fig. 6. The FP binding assay data. (a) FP data for Pf $(100 \mathrm{nM}), \mathrm{R} 6 \mathrm{G}$ and Et (each $1 \mu \mathrm{M})$ in the presence of StAcrR and human ENO1. (b) Measuring FP with a range of concentrations of Pf. Red error bars represent standard deviation from the mean value of independent measurements 
for each of the StAcrR and ENO1 (not applicable for panel $b$ ) concentrations. Black curves are sigmoidal fittings for each of the ligands.

Fig. 7. Loop-to-helix transition at the multidrug-binding site. Variations in the main-chain and side-chain conformations of Gln14, Asp18, Glu67, Arg105, Arg106 and Gln130 in StAcrR (orange), EcAcrR-P222 ${ }_{1}$ (cyan) and EcAcrR-P3 ${ }_{1}$ (magenta) are shown. 


\section{Table 1}

$\mathrm{X}$-ray data-collection and refinement statistics.

Values in parentheses are for the highest resolution shell.

Data-collection statistics

X-ray source

APS 19-ID beamline

Wavelength (̊)

0.9792

Resolution range $(\AA)$

37.9-1.56 (1.59-1.56)

Space group

$P 2_{1}$

Cell dimensions

$a, b, c(\AA)$

$47.18,75.82,55.8$

$\alpha, \beta, \gamma\left(^{\circ}\right)$

$90.0,108.7,90.0$

Unique reflections

$51,039(2,219)$

Completeness (\%)

$97.5(85.1)$

$R_{\text {merge }}(\%)$

$5.4(75.9)$

$R_{\text {meas }}(\%)$

$5.9(84.4)$

$I / \sigma I$

$9.3(2.12)$

Multiplicity

$5.1(4.3)$

$\mathrm{CC} 1 / 2$

N/A $(0.7)$

Refinement statistics

PDB ID

3 lhq

Resolution range ( $\mathrm{A})$

$37.9-1.56(1.60-1.56)$

$R_{\text {work }}(\%)$

$15.0(26.4)$

$R_{\text {free }}(\%)$

$19.9(35.2)$

No. atoms

Protein

Chain A

1,812

Chain $B$

1,840 


\begin{tabular}{ll}
\hline Water & 281 \\
1,2-ethanediol & 4 \\
di(hydroxyethyl)ether & 14 \\
Average $B\left(\AA^{2}\right)$ & \\
Protein & 18.2 \\
Chain $A$ & 18.9 \\
Chain $B$ & 35.2 \\
Water & 31.6 \\
1,2-ethanediol & 36.3 \\
di(hydroxyethyl)ether & \\
R.m.s. deviations & 0.02 \\
Bond lengths $(\AA)$ & 1.64 \\
Bond angles ( $\left.{ }^{\circ}\right)$ & \\
Ramachandran plot & \\
Favoured $(\%)$ & 0.0 \\
Allowed $(\%)$ & 0.0 \\
Outliers $(\%)$ &
\end{tabular}

${ }^{\mathrm{a}}$ Ramachandran statistics are based on the PDB and MolProbity (Chen et al., 2010) validation reports. 


\section{Table 2}

Competition and binding FP data for StAcrR.

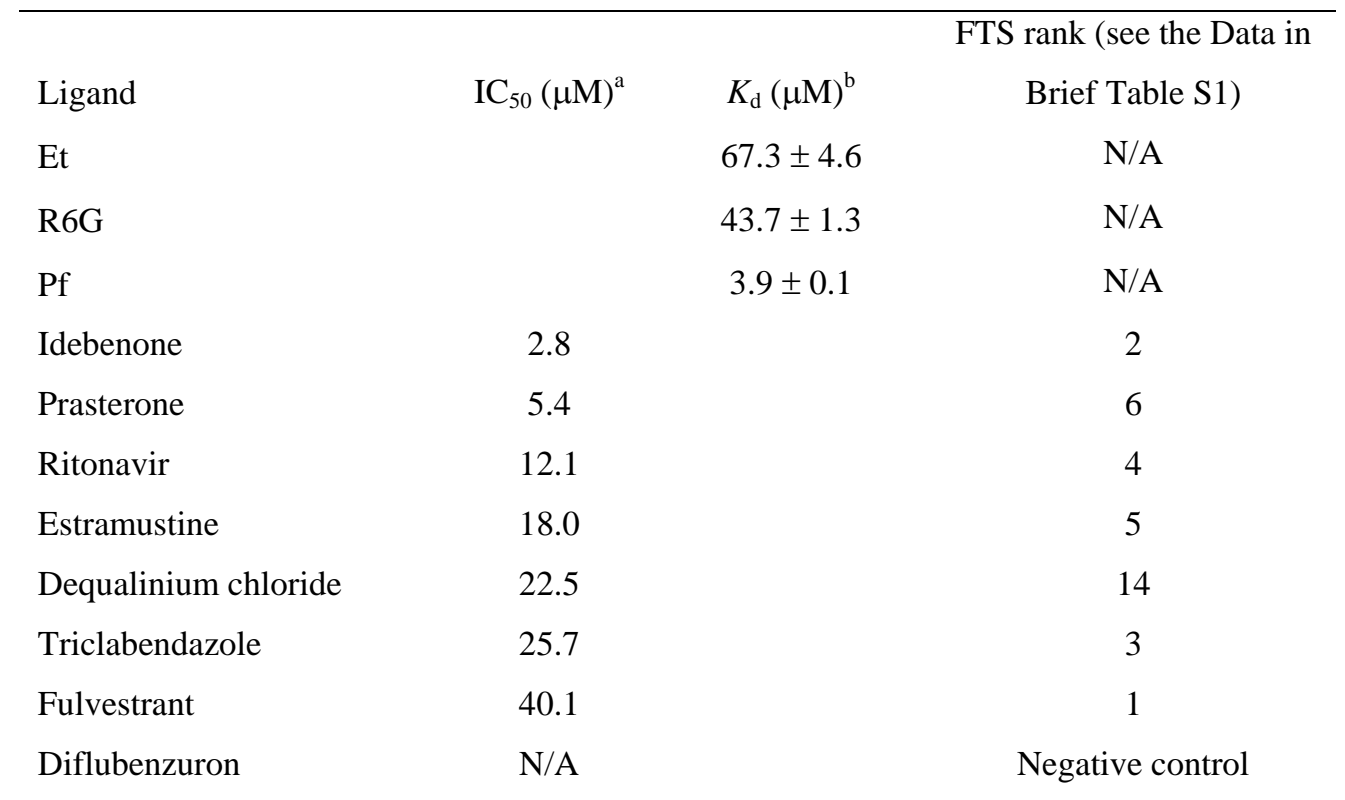

${ }^{\mathrm{a}}$ Values obtained in the presence of $10 \mu \mathrm{M}$ StAcrR and $100 \mathrm{nM}$ Pf.

${ }^{\mathrm{b}}$ Values obtained in the presence of $100 \mathrm{nM}$ Pf, $1 \mu \mathrm{M}$ Et and $1 \mu \mathrm{M}$ R6G. 


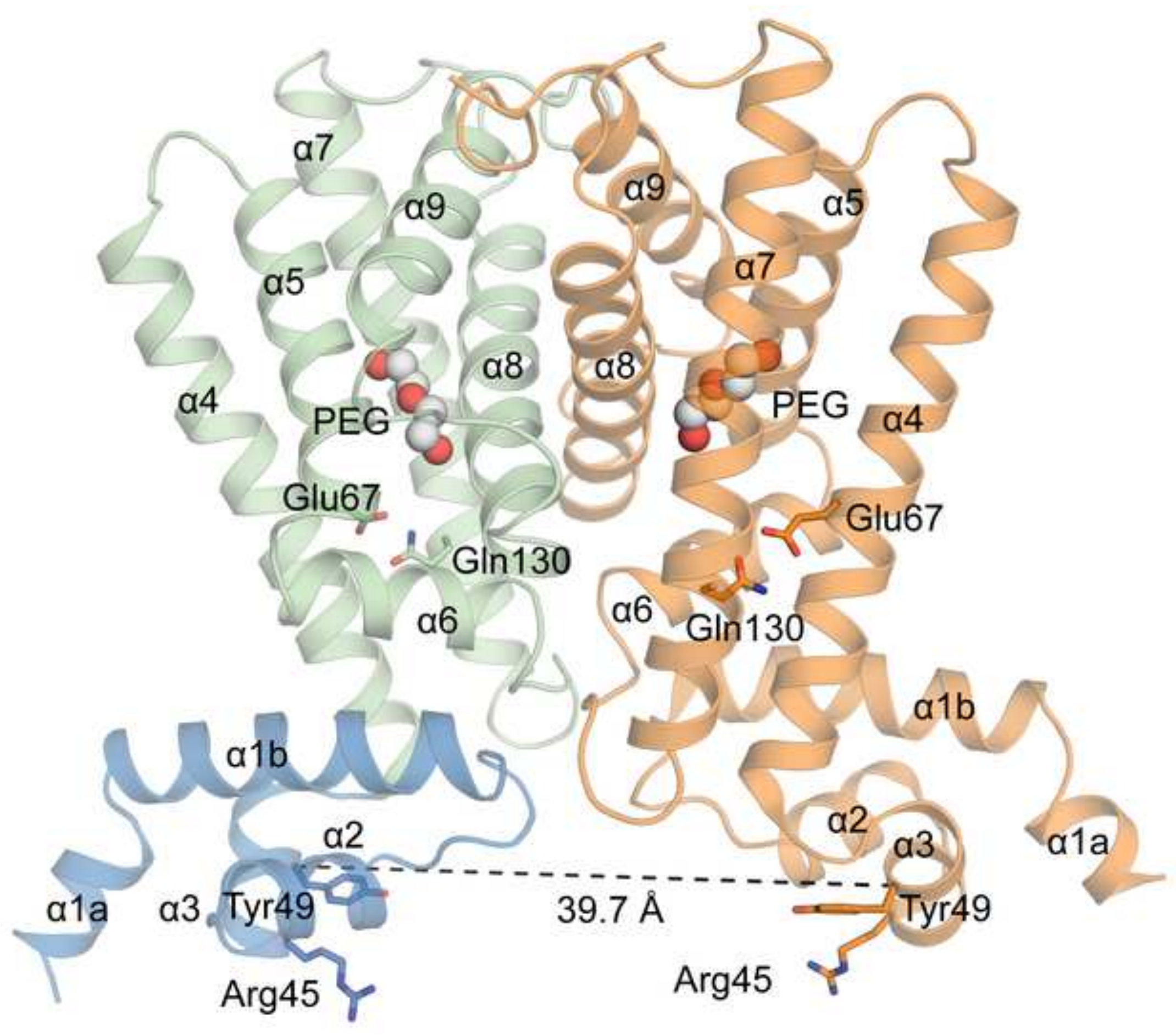


(a)

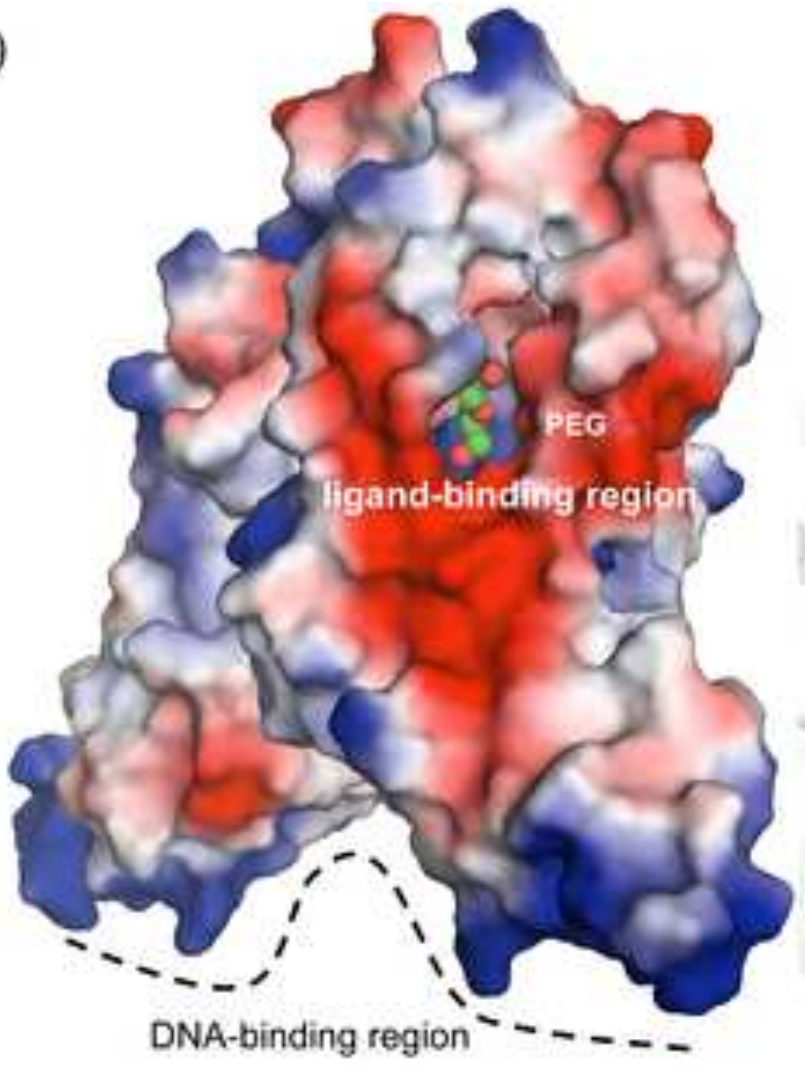

(b)

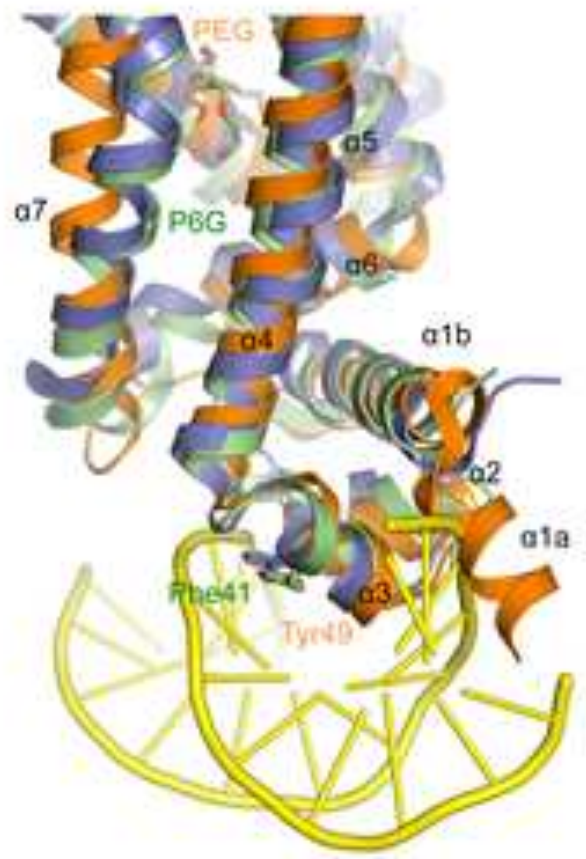

(c)

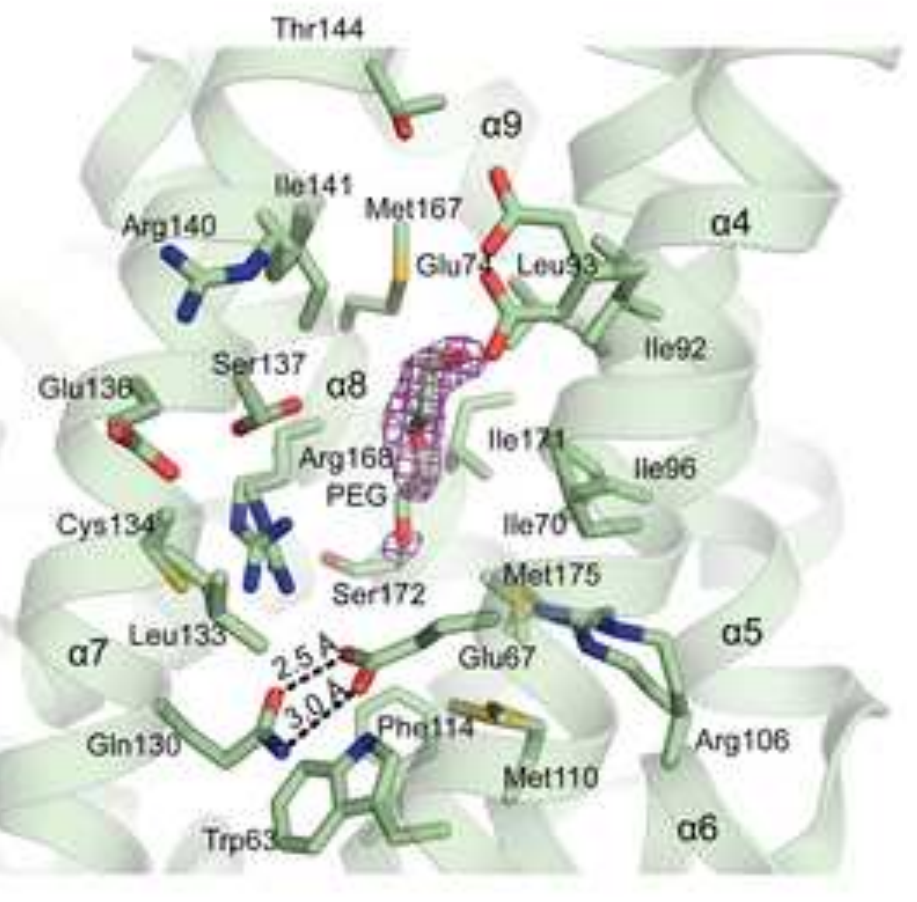

Typhinuriun

T. maritima

$|\boldsymbol{E} .0011|$

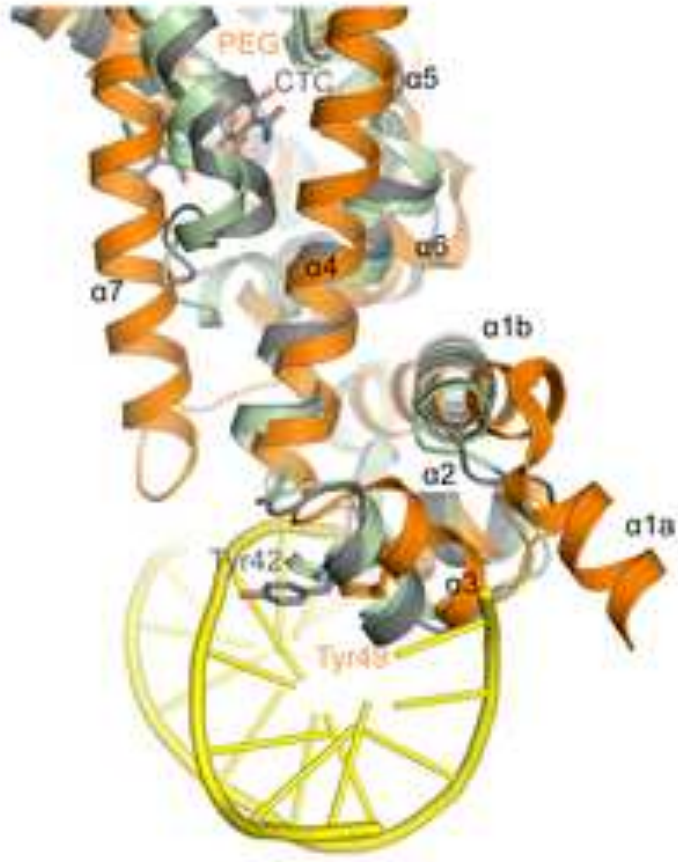

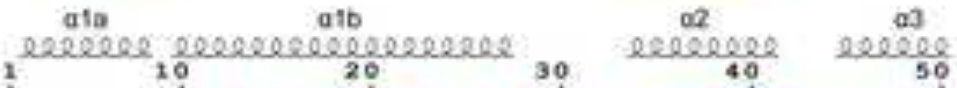

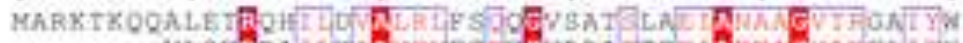

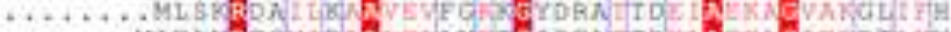

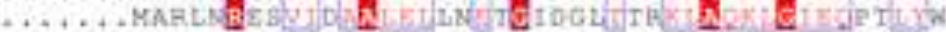

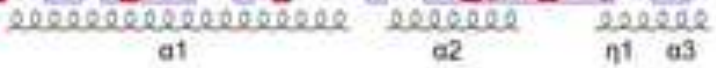

Q4 as

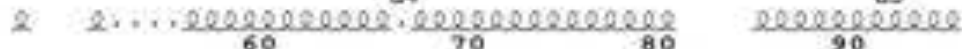

|8. typhimuriun | |T.maritima|

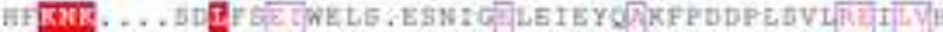

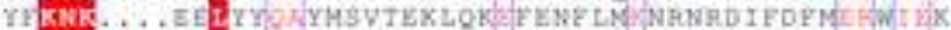

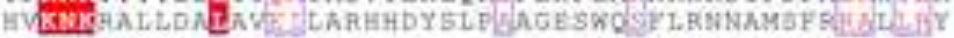


(a)

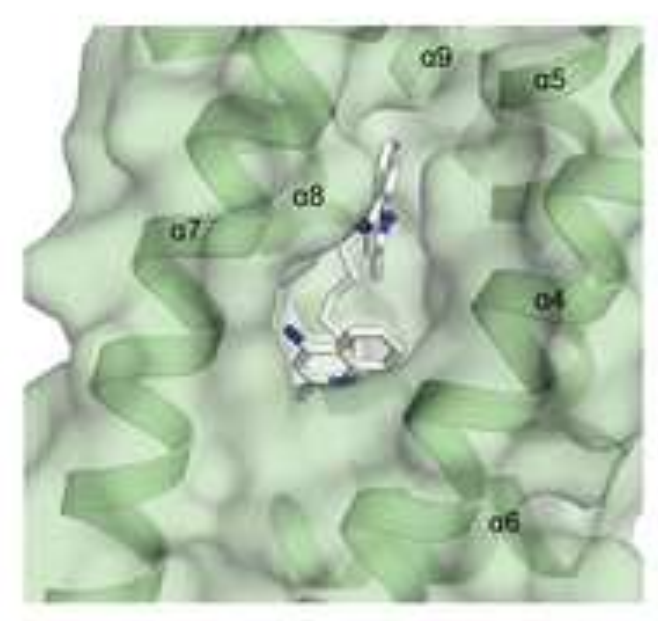

(b)

\section{Ethidium}
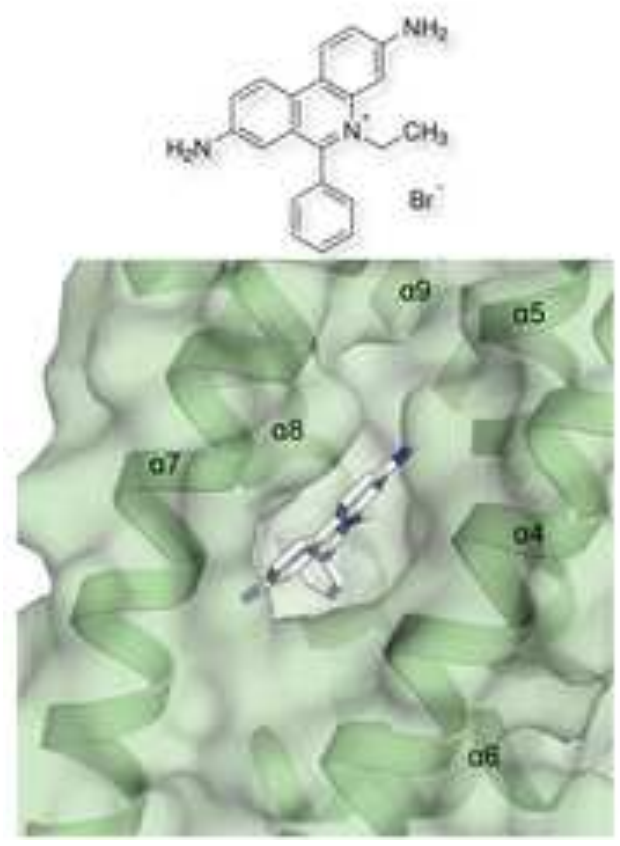

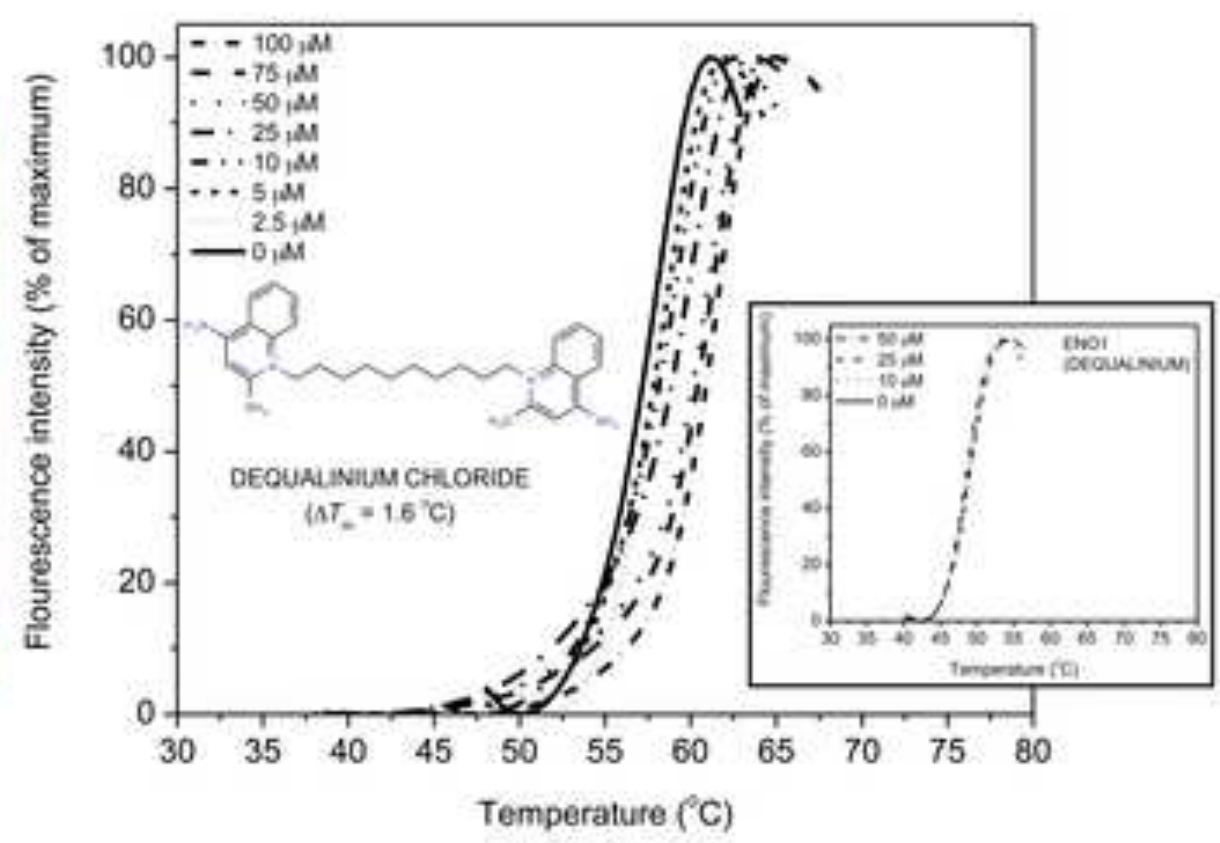

(c)

(d)

Proflavine
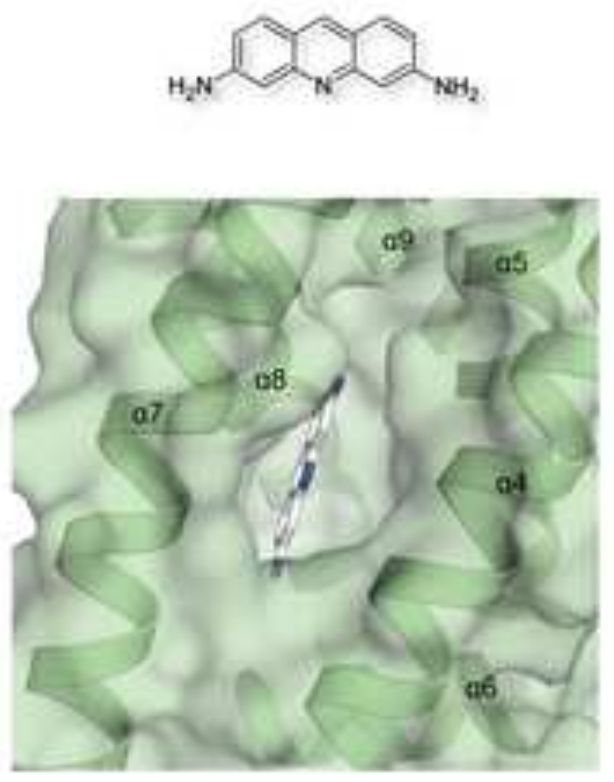

\section{Rhodamine 6G}

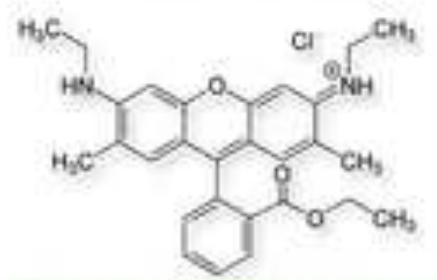

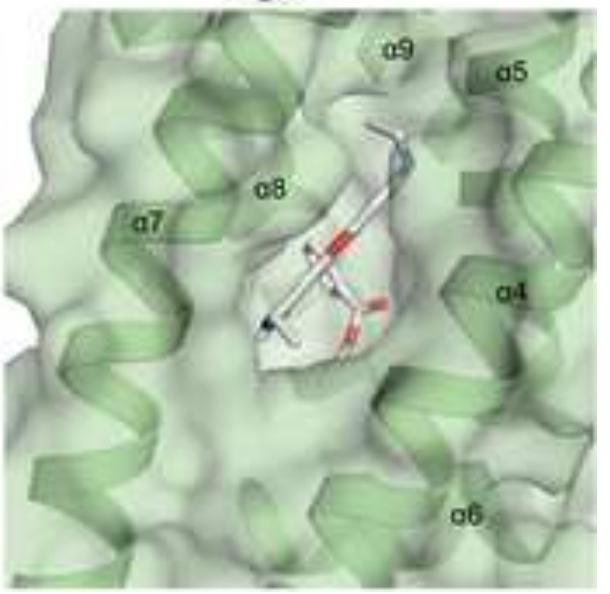



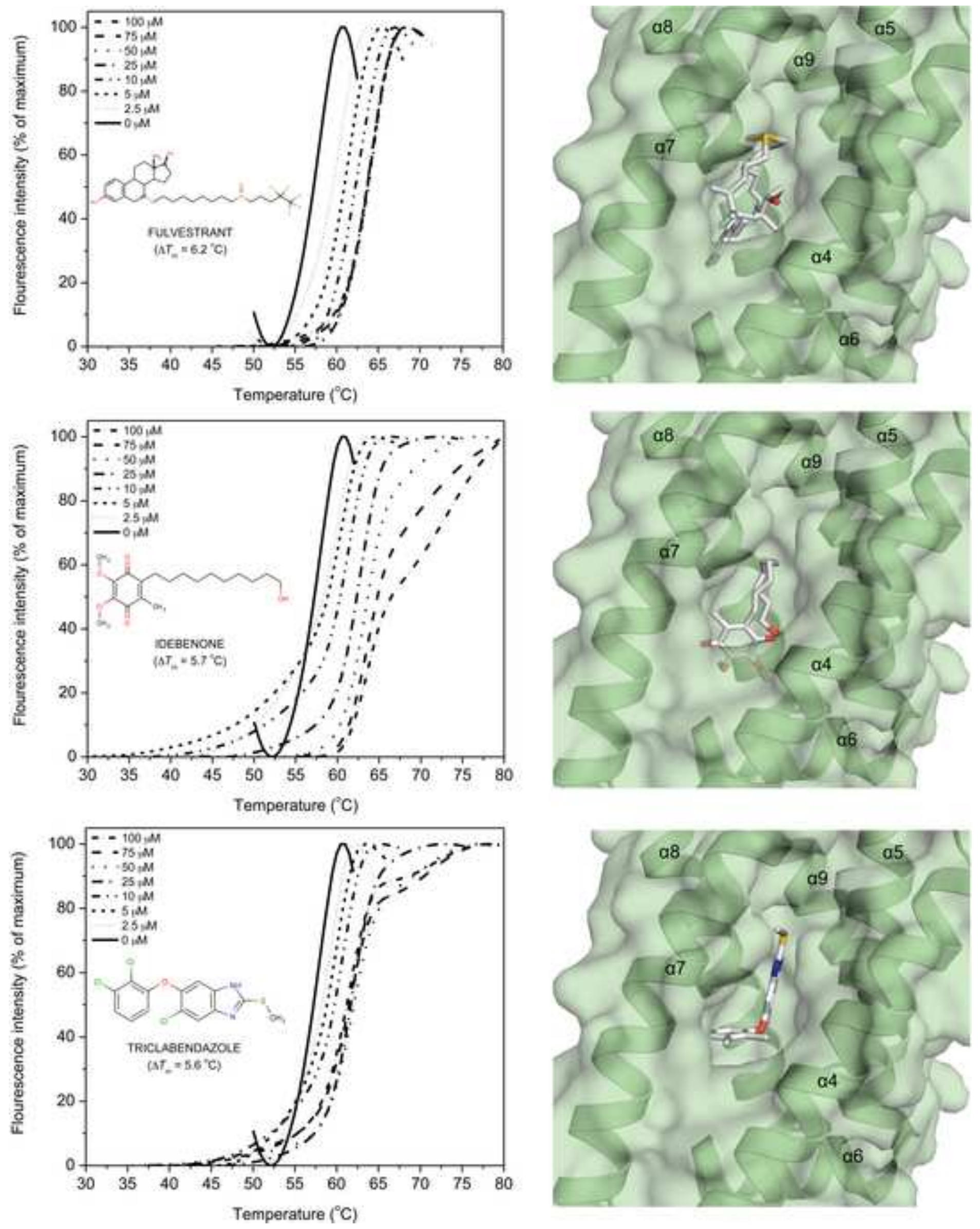

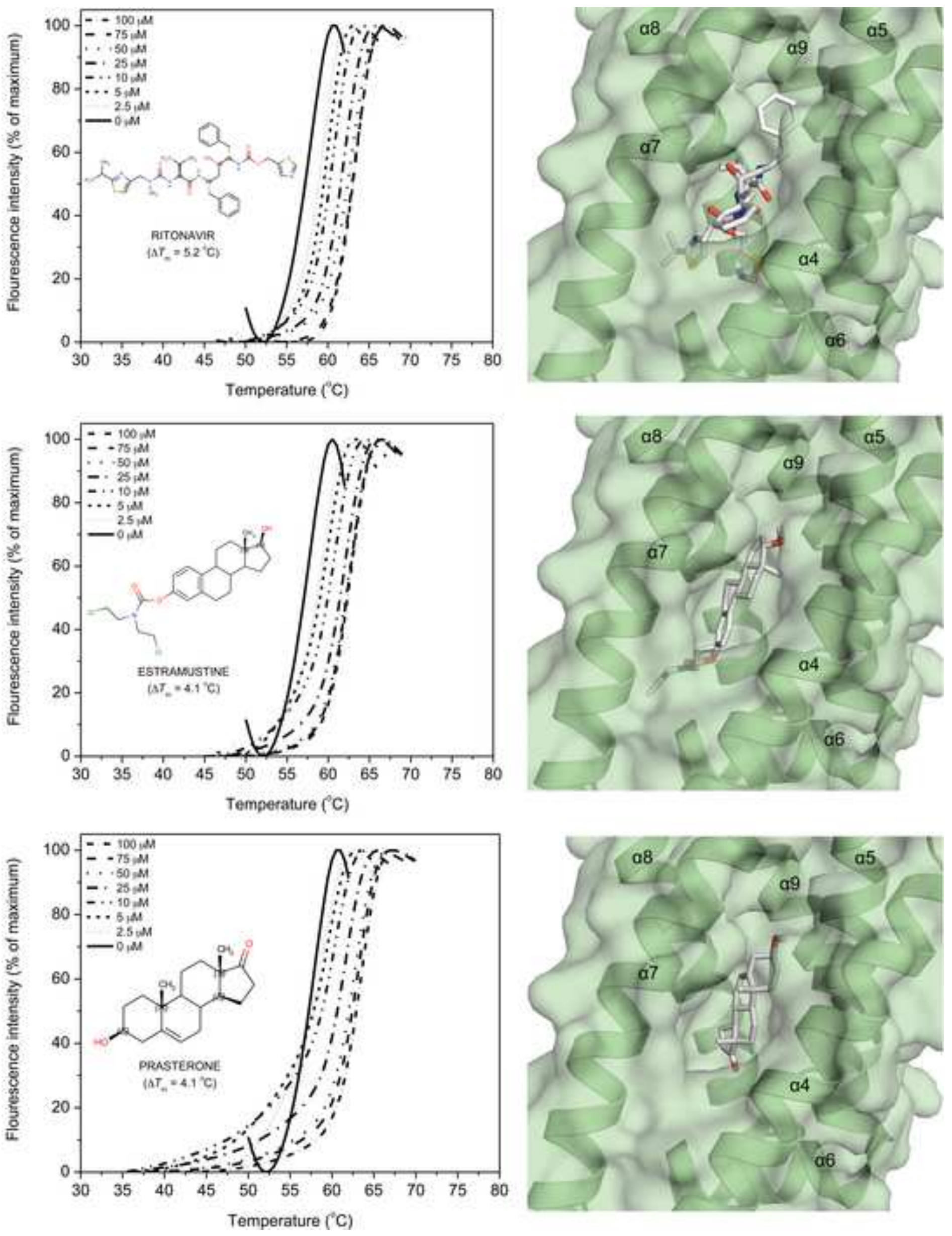
(a)

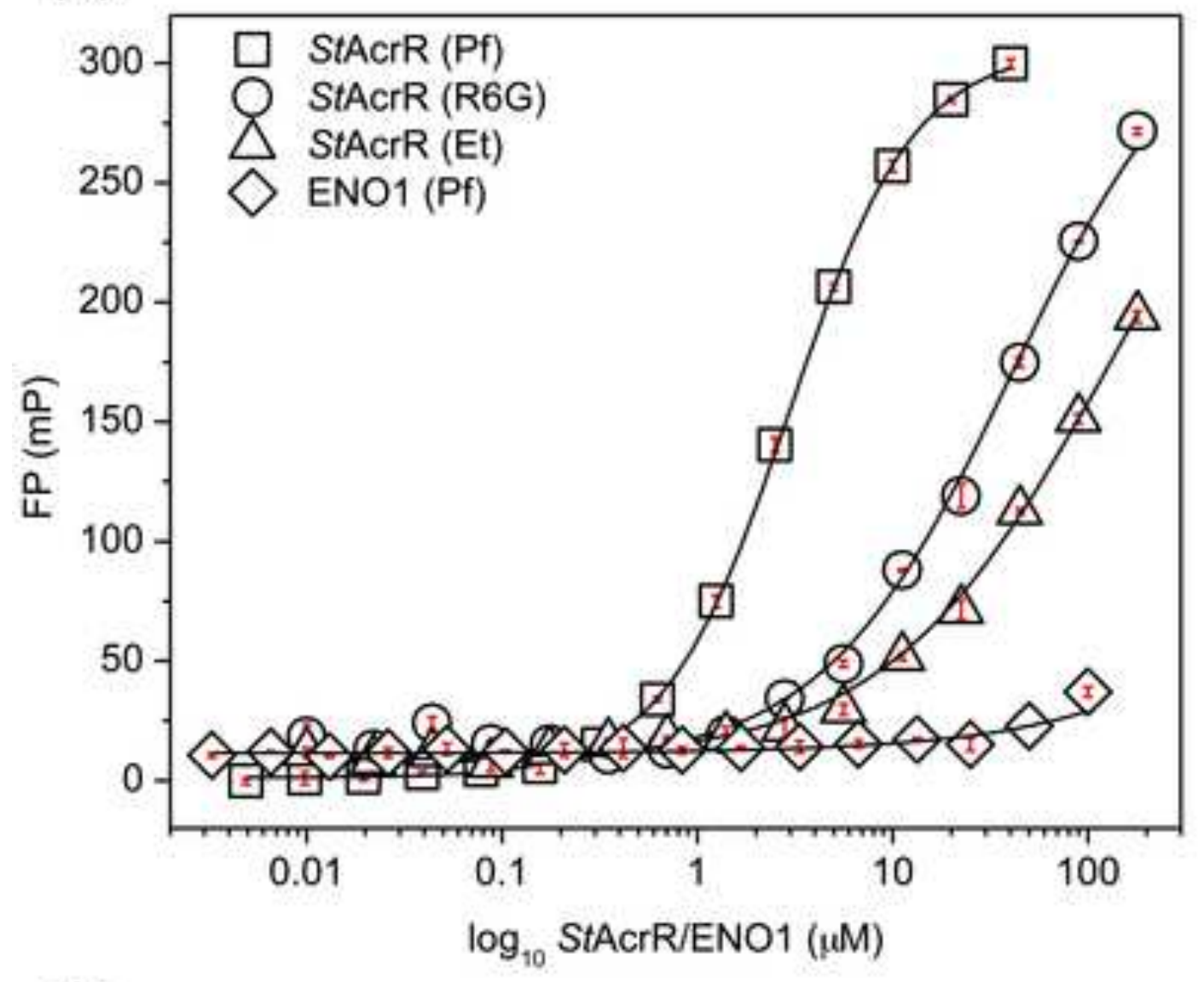

(b)

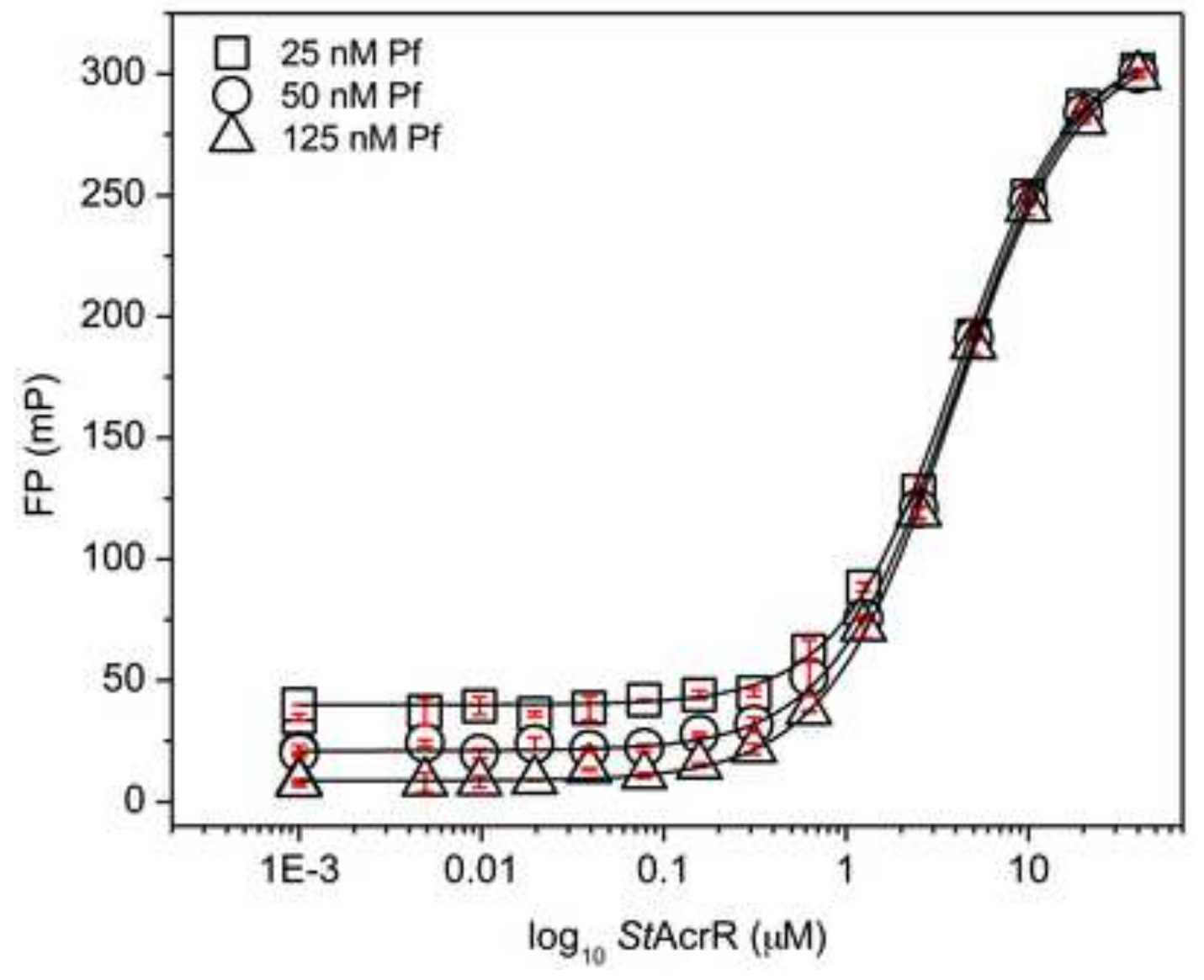




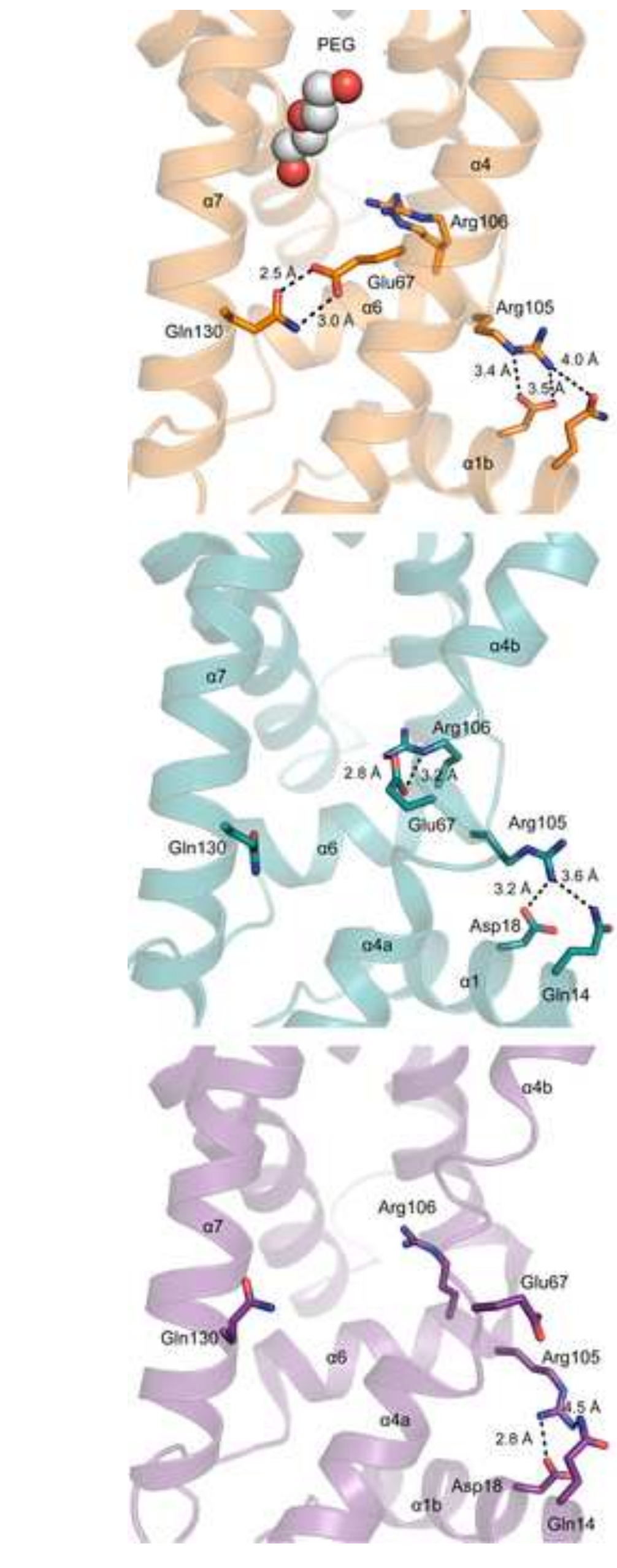

Figure

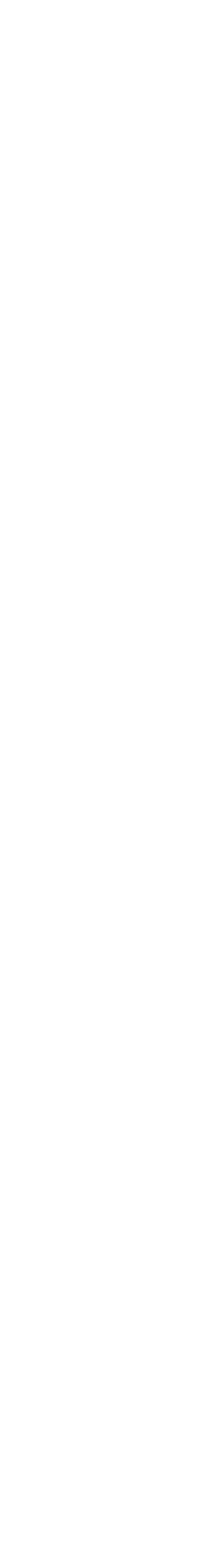

